\title{
Itération de polynômes et fonctions entières arithmétiques
}

\author{
par \\ Jean-Paul BÉzivin (Caen)
}

I. Introduction. Soit $f(z)$ une fonction entière d'une variable complexe. On dit que $f(z)$ est arithmétique au sens de Pólya, si on a $f(n) \in \mathbb{Z}$ pour tout $n \in \mathbb{N}$.

Un théorème de Pólya montre que si la croissance de $f$ n'est pas trop grande, $f(z)$ est polynômiale $([\mathrm{PO}])$ :

ThÉORÈme P. Soit $f(z)$ une fonction entière d'une variable complexe. On pose $|f|(R)=\max (|f(z)|: z \in \mathbb{C},|z| \leq R)$. Si on a, pour tout $R$ assez grand,

$$
\log |f|(R) \leq \delta R
$$

avec $\delta<\log 2$, alors $f(z)$ est un polynôme.

Soit $q$ un nombre entier naturel, avec $|q|>1$. On suppose que la fonction $f(z)$ est telle que $f\left(q^{n}\right) \in \mathbb{Z}$ pour tout $n \in \mathbb{N}$. Un théorème de Gel'fond montre que, si la croissance de $f(z)$ n'est pas trop grande, $f(z)$ est un polynôme ([GU], théorème VIII, p. 179) :

ThÉORÈME G. Soit $f(z)$ une fonction entière d'une variable complexe $z$. Soit $q \in \mathbb{Z},|q|>1$. On suppose que $f\left(q^{n}\right) \in \mathbb{Z}$ pour tout $n \in \mathbb{N}$, et que l'on a

$$
\log |f|(R) \leq \frac{(\log R)^{2}}{4 \log |q|}-\frac{\log R}{2}-\omega(R)
$$

pour tout $R$ assez grand, où $\omega(R)$ est une fonction tendant vers l'infini si $R$ tend vers l'infini. Alors $f(z)$ est un polynôme.

Dans cet article, nous allons obtenir une généralisation des deux résultats que nous venons de citer.

Soit $P$ un polynôme à coefficients dans $\mathbb{Z}$, que nous supposerons dans tout le reste de l'article de degré $\geq 1$. Pour $n \in \mathbb{N}, n \geq 1$, on note $P^{[n]}$ le composé de $P(x) n$ fois avec lui-même, et par convention $P^{[0]}(x)=x$. Soit $f(z)$ une fonction entière d'une variable complexe, et $z \in \mathbb{Z}$. Nous supposons 
que

$$
f\left(P^{[n]}(z)\right) \in \mathbb{Z}
$$

pour tout $n \in \mathbb{Z}$. On se propose de donner des conditions sur la croissance de $f$ pour que cette condition implique que $f$ est un polynôme.

Pour voir le rapport avec les théorèmes $\mathrm{P}$ et $\mathrm{G}$, nous examinons deux cas particuliers.

Dans le cas où le polynôme $P$ est égal à $x+1$, on voit que $P^{[n]}(x)=x+n$. Il en résulte que pour la valeur $z=0$, les fonctions arithmétiques relative à ce polynôme et ce point sont les fonctions arithmétiques au sens de Pólya.

Dans le cas où le polynôme $P$ est égal à $q x$, où $q$ est un élément de $\mathbb{Z}$ tel que $|q|>1$, on voit que $P^{[n]}(x)=q^{n} x$. Il en résulte que, pour le choix de $z=1$, les fonctions arithmétiques relative à ce polynôme et ce point sont les fonctions arithmétiques au sens de Gel'fond.

Il est facile de voir que le cas où le polynôme $P$ est de degré 1 se ramène en fait à l'un ou l'autre de ces deux cas.

Nous ferons désormais l'hypothèse que le degré de $P$ est plus grand que 1 , sauf mention expresse du contraire.

Nous ferons dans tout l'article l'hypothèse que le point $z$ est choisi de façon que pour $k, j \in \mathbb{N}$, avec $k \neq j$, on ait $P^{[k]}(z) \neq P^{[j]}(z)$.

Nous notons le degré du polynôme $P$ par $d$, et $L=L(z, P)$ la fonction de Green du bassin d'attraction du point à l'infini pour le polynôme $P$ (cf. [BEGEMO]).

Il est facile de voir que la condition précédente sur $z$ est vérifiée pour $|z|$ assez grand. Il résultera du lemme 2.10 qu'il faut et il suffit pour qu'il en soit ainsi que le point $z$ ne soit pas dans l'ensemble de Julia rempli $K_{P}$ associé à $P$.

Notre but principal est le résultat suivant :

ThÉORÈme 1. Soit $f(z)$ une fonction entière d'une variable complexe, et $P \in \mathbb{Z}[x]$, de degré $d \geq 2, z \in \mathbb{Z}$ vérifiant les hypothèses précédentes. On suppose que $f\left(P^{[n]}(z)\right) \in \mathbb{Z}$ pour tout $n \in \mathbb{Z}$ et de plus que l'on a la majoration

$$
\log |f|(R) \leq \frac{\log R \log \log R-\log R \log \log \log R}{\log d}-\lambda \log R
$$

pour tout $R$ assez grand, avec

$$
\lambda>\frac{L}{d}-\frac{\log \log d}{\log d} .
$$

Alors $f$ est un polynôme.

La démonstration utilise la méthode de la série d'interpolation, qui est à la base des démonstrations des théorèmes $\mathrm{P}$ et $\mathrm{G}$. 
Nous montrerons de plus que le résultat, comme ceux des théorèmes $\mathrm{P}$ et $\mathrm{G}$, est le meilleur possible :

Proposition 2. La fonction

$$
f(x)=\sum_{k=0}^{\infty} \frac{\prod_{j=0}^{k-1}\left(x-P^{[j]}(z)\right)}{\prod_{j=0}^{k-1}\left(P^{[k]}(z)-P^{[j]}(z)\right)}
$$

est une fonction entière de la variable complexe $x$, non polynômiale, telle que

$$
\log |f|(R) \leq \frac{\log R \log \log R-\log R \log \log \log R}{\log d}+O(\log R)
$$

pour tout $R$ assez grand, et vérifie $f\left(P^{[n]}(z)\right) \in \mathbb{Z}$ pour tout $n \in \mathbb{N}$.

Corollaire 3. Soit $d \in \mathbb{Z}, d \geq 2$ et $z \in \mathbb{Z},|z|>1$. Soit $f(x)$ une fonction entière de la variable complexe $x$. On suppose que $f\left(z^{d^{n}}\right) \in \mathbb{Z}$ pour tout $n \in \mathbb{N}$, et que $f$ vérifie l'estimation

$$
\log |f|(R) \leq \frac{\log R \log \log R-\log R \log \log \log R}{\log d}-\lambda \log R
$$

pour tout $R$ assez grand, avec

$$
\lambda>\frac{\log |z|}{d}-\frac{\log \log d}{\log d} .
$$

Alors $f$ est un polynôme.

Corollaire 4. Soit $z \in \mathbb{Z},|z| \geq 3$. Soit $\omega$ la racine de module plus grand que 1 de l'équation $X^{2}-(z / 2) X+1=0$. Soit $f(x)$ une fonction entière d'une variable complexe telle que $f\left(\omega^{2^{n}}+\omega^{-2^{n}}\right) \in \mathbb{Z}$ pour tout $n \in \mathbb{N}$. On suppose de plus que $f$ vérifie l'estimation

$$
\log |f|(R) \leq \frac{\log R \log \log R-\log R \log \log \log R}{\log 2}-\lambda \log R
$$

pour tout $R$ assez grand, avec

$$
\lambda>\frac{\log \left|\frac{z+\sqrt{z^{2}-4}}{2}\right|}{2}-\frac{\log \log 2}{\log 2} .
$$

Alors $f$ est un polynôme.

R e marque. Dans tout le texte, un produit sur un ensemble vide d'indices sera pris égal à 1 , et une somme sur un ensemble vide d'indices à zéro.

II. Lemmes préliminaires. Soit $A$ un anneau commutatif intègre unitaire, et $P \in A[x]$ un polynôme de degré $d \geq 1$. Soit $n$ un entier naturel et 
$k \in\{0, \ldots, n\}$. On pose

$$
Q_{k, n}(x)=\prod_{0 \leq j \leq n, j \neq k}\left(P^{[j]}(x)-P^{[k]}(x)\right) .
$$

Il est facile de voir que le polynôme $Q_{k, n}(x)$ ne peut être nul que si $P$ est de la forme $P(x)=x$ ou $P(x)=a x+b$ avec $a$ racine de l'unité différente de 1 et $b$ quelconque (auquel cas on a $P^{[n]}(x)=x$ si $a^{n}=1$ ). Nous supposerons dans toute la suite que $P$ n'est pas de cette forme. On a alors le résultat suivant :

Proposition 2.1. Tous les polynômes $Q_{k, n}(x), k=0, \ldots, n$, divisent le polynôme $Q_{n, n}(x)$ dans $A[x]$.

Remarque. La proposition 2.1 ci-dessus nous paraît avoir un intérêt propre. Elle contient comme cas particuliers les lemmes de nature analogue utilisés dans les démonstrations des théorèmes $\mathrm{P}$ et $\mathrm{G}$.

Le démonstration de la proposition 2.1 résultera des lemmes qui suivent.

Nous supposons tout d'abord que le degré $d$ de $P$ est supérieur ou égal à 2 , et nous éliminerons cette restriction à la fin de la démonstration. Pour les lemmes 2.2 à 2.6, l'anneau $A$ est pris égal à $\mathbb{C}$.

LEMME 2.2. Soit $\omega$ un nombre complexe. On suppose qu'il existe un entier non nul $m$ tel que $P^{[m]}(\omega)=\omega$. Alors il existe un plus petit entier $l=l(\omega) \geq 1$ tel que l'on ait $P^{[l]}(\omega)=\omega$; nous dirons que $l(\omega)$ est l'ordre de $\omega$. De plus, une égalité $P^{[m]}(\omega)=\omega$ avec $m \geq 1$ implique que l divise $m$.

Démonstration. Seule est à démontrer la seconde affirmation, la première étant claire. Posons $m=k l+r$ avec $0 \leq r<l$. On a immédiatement que $P^{[r]}(\omega)=\omega$, et la définition de $l(\omega)$ implique alors $r=0$ et le résultat.

Lemme 2.3. Soit $z \in \mathbb{C}$ et $k \in \mathbb{N}$. On suppose que $\omega_{k}=P^{[k]}(z)$ a un ordre. Les indices $j \in \mathbb{N}$ tels que $P^{[k]}(z)=P^{[j]}(z)$ sont de la forme $j=j_{0}+h l\left(\omega_{k}\right)$, où $j_{0}$ est le plus petit d'entre eux, et $h \in \mathbb{N}$.

Démonstration. Il est clair que si $j_{0}$ est un entier tel que $P^{[k]}(z)=$ $P^{\left[j_{0}\right]}(z)$, alors tout entier $j$ de la forme $j=j_{0}+h l$ vérifie aussi une telle égalité. Réciproquement, soit $j$ un entier tel que $P^{[k]}(z)=P^{[j]}(z)$; posons $j-j_{0}=h l+r$ avec $0 \leq r<l$. On a $h \in \mathbb{N}$ en raison de la définition de $j_{0}$, de sorte que

$$
P^{[j]}(z)=P^{[r]}\left(P^{[h l]}\left(P^{\left[j_{0}\right]}(z)\right)\right)=P^{[r]}\left(P^{[h l]}\left(P^{[k]}(z)\right)\right)=P^{[r]}\left(P^{\left[j_{0}\right]}(z)\right) .
$$

Comme $P^{[j]}(z)=P^{\left[j_{0}\right]}(z)=P^{[k]}(z)$, il vient $P^{[r]}\left(\omega_{k}\right)=\omega_{k}$, et donc $r=0$ d'après la définition de $l\left(\omega_{k}\right)$.

Lemme 2.4. Soit $z \in \mathbb{C}$ fixé. On suppose qu'il existe un entier $N \geq 1$ tel que $\omega_{N}=P^{[N]}(z)$ ait un ordre. Alors la fonction $k \rightarrow l\left(P^{[k]}(z)\right)$ est définie et décroissante pour $k \geq N$. 
Démonstration. Soit $l=l\left(\omega_{N}\right)$. On a donc $P^{[l]}\left(\omega_{N}\right)=\omega_{N}$, et par suite, en composant avec un polynôme $P^{[h]}$ il vient $P^{[l]}\left(\omega_{N+h}\right)=\omega_{N+h}$; ceci démontre que $\omega_{N+h}$ a un ordre et que celui-ci divise $l$ d'après le lemme 2.2 , d'où le résultat.

Lemme 2.5. Soit $z \in \mathbb{C}$. On note $M(z, k)$ la multiplicité de la racine $z$ dans le polynôme $P^{[k]}(x)-P^{[k]}(z)$. Alors $M(z, k)$ est une fonction croissante de $k$.

Démonstration. On peut écrire

$$
P(U)-P(V)=(U-V) Q(U, V)
$$

où $Q(U, V)$ est un polynôme des deux variables $U, V$. On en déduit que

$$
P^{[k+1]}(x)-P^{[k+1]}(z)=\left(P^{[k]}(x)-P^{[k]}(z)\right) Q\left(P^{[k]}(x), P^{[k]}(z)\right)
$$

et le lemme en résulte.

LEMME 2.6. Le résultat de la proposition 2.1 est vrai dans le cas où $A=\mathbb{C}$, et sous l'hypothèse supplémentaire que tous les polynômes $P^{[k]}(x)-x$, $k \in\{1, \ldots, n\}$, n'aient pas de racines multiples dans $\mathbb{C}$.

Démonstration. On fixe un entier $n$ et un entier $k$ tel que $0 \leq k \leq n$. On peut clairement supposer que $k<n$. Soit $z \in \mathbb{C}$ une racine de $Q_{k, n}(x)$. Alors il existe un indice $j$ tel que $P^{[k]}(z)=P^{[j]}(z)$ avec $j \neq k$. En composant par un polynôme de la forme $P^{[h]}(x)$ pour un $h$ convenable, on montre qu'il existe un entier $j^{\prime}<n$ tel que $P^{[n]}(z)=P^{\left[j^{\prime}\right]}(z)$, donc $z$ est racine du polynôme $Q_{n, n}(x)$.

Nous allons maintenant démontrer que la multiplicité de $z$ dans $Q_{k, n}(x)$ est inférieure ou égale à la multiplicité de $z$ dans $Q_{n, n}(x)$.

Considérons tout d'abord un indice $j$ tel que $0 \leq j<k$, avec $P^{[k]}(z)=$ $P^{[j]}(z)$. On écrit

$$
P^{[k]}(x)-P^{[j]}(x)=P^{[k-j]}\left(P^{[j]}(x)\right)-P^{[j]}(x) .
$$

Le polynôme $P^{[k-j]}(x)-x$ n'ayant que des racines simples, il en résulte que la multiplicité de $z$ dans le polynôme $P^{[k]}(x)-P^{[j]}(x)$ est égale à la multiplicité de $z$ dans le polynôme $P^{[j]}(x)-P^{[j]}(z)$.

Un raisonnement analogue montre que la multiplicité de $z$ dans $P^{[k]}(x)-$ $P^{[j]}(x)$ quand $j>k$ est égale à celle de $z$ dans $P^{[k]}(x)-P^{[k]}(z)$.

On rappelle que $M(z, m)$ est la multiplicité de $z$ dans $P^{[m]}(x)-P^{[m]}(z)$. D'après ce qui précède, la multiplicité de $z$ dans $Q_{k, n}(x)$ est

$$
\sum_{0 \leq j<k, P^{[k]}(z)=P^{[j]}(z)} M(z, j)+\sum_{k<j \leq n, P^{[k]}(z)=P^{[j]}(z)} M(z, k),
$$


tandis que la multiplicité de $z$ dans $Q_{n, n}(x)$ est

$$
\sum_{0 \leq j^{\prime}<n, P^{[n]}(z)=P^{\left[j^{\prime}\right]}(z)} M\left(z, j^{\prime}\right) .
$$

Considérons un entier $j$ tel que $k<j \leq n$ et $P^{[k]}(z)=P^{[j]}(z)$. En composant avec $P^{[n-j]}(x)$, il vient $P^{[n]}(z)=P^{[n-j+k]}(z)$. Posons $j^{\prime}=n-j+k$. On a $j^{\prime} \geq k$ car $n \geq j$, et $j^{\prime}<n$ car $j>k$.

Le lemme 2.5 montre que $M(z, k) \leq M\left(z, j^{\prime}\right)$, donc

$$
\sum_{k<j \leq n, P^{[k]}(z)=P^{[j]}(z)} M(z, k) \leq \sum_{k \leq j^{\prime}<n, P^{[n]}(z)=P^{\left[j^{\prime}\right]}(z)} M\left(z, j^{\prime}\right) .
$$

Il nous reste à démontrer que

$$
\sum_{0 \leq j<k, P^{[k]}(z)=P^{[j]}(z)} M(z, j) \leq \sum_{0 \leq j^{\prime}<k, P^{[n]}(z)=P^{\left[j^{\prime}\right]}(z)} M\left(z, j^{\prime}\right) .
$$

Soit $j_{0}$ le premier indice $\geq 0$ tel que $P^{[j]}(z)=P^{[k]}(z)$. Si $j_{0}=k$, il n'y a rien à démontrer, de sorte que nous pouvons supposer $j_{0}<k$. Le lemme 2.3 montre que les entiers $j$ sur lesquels se fait la première sommation sont les entiers $j$ de la forme $j=j_{0}+h l$ avec $l=l\left(P^{[k]}(z)\right)$ et $0 \leq h<\left(k-j_{0}\right) / l \in \mathbb{N}$. Posons $n-j_{0}=\alpha l+\beta$ avec $\alpha \in \mathbb{N}$ et $\beta \in \mathbb{N}, 0 \leq \beta<l$. On a alors

$$
P^{[n]}(z)=P^{\left[j_{0}+\alpha l+\beta\right]}(z)=P^{[\beta]}\left(P^{[\alpha l]}\left(P^{\left[j_{0}\right]}(z)\right)\right)=P^{\left[j_{0}+\beta\right]}(z),
$$

ce qui montre que $j_{0}^{\prime}=j_{0}+\beta$ est un indice tel que $P^{\left[j_{0}^{\prime}\right]}(z)=P^{[n]}(z)$. Pour $h<\left(k-j_{0}\right) / l$, on pose $j^{\prime}=j_{0}^{\prime}+h l$. On a $j^{\prime} \geq 0, j^{\prime} \geq j=j_{0}+h l$, et d'autre part,

$$
j^{\prime}=j_{0}+\beta+h l \leq j_{0}+\beta+\left(\frac{k-j_{0}}{l}-1\right) l=k+\beta-l<k .
$$

Enfin, on a

$$
P^{\left[j^{\prime}\right]}(z)=P^{\left[j_{0}+\beta+h l\right]}(z)=P^{[\beta]}\left(P^{[h l]}\left(P^{\left[j_{0}\right]}(z)\right)\right)=P^{\left[\beta+j_{0}\right]}(z)=P^{[n]}(z) .
$$

En utilisant encore la croissance de la fonction $m \rightarrow M(z, m)$, il en résulte

$$
\sum_{0 \leq j<k, P^{[k]}(z)=P^{[j]}(z)} M(z, j) \leq \sum_{0 \leq j^{\prime}<k, P^{[n]}(z)=P^{\left[j^{\prime}\right]}(z)} M\left(z, j^{\prime}\right),
$$

ce qui termine la démonstration du lemme.

Nous fixons un entier $d \geq 2$, et nous considérons dans $\mathbb{Z}\left[t_{0}, \ldots, t_{d}, x\right]$ le polynôme $P(x)=t_{d} x^{d}+\ldots+t_{0}$.

Lemme 2.7. Pour $k \in \mathbb{N}, k \geq 1$, le discriminant de $P^{[k]}(x)-x$ est une fonction polynômiale de $t_{0}, \ldots, t_{d}$, qui n'est pas identiquement nulle. 
Démonstration. Le fait que le discriminant en question soit une fonction polynômiale de $t_{0}, \ldots, t_{d}$ vient du fait que le discriminant d'un polynôme est fonction polynômiale de ses coefficients, et que les coefficients de $P^{[k]}(x)-x$ sont des fonctions polynômiales de $t_{0}, \ldots, t_{d}$.

Pour prouver que ce discriminant est un polynôme non nul, il suffit de donner un exemple. Posons $P(x)=(1+x)^{d}-1$. Ce polynôme est de degré exactement $d$, et on a la formule

$$
P^{[k]}(x)=(1+x)^{d^{k}}-1 .
$$

Un calcul immédiat montre que $P^{[k]}(x)-x$ n'a pas de racines multiples dans $\mathbb{C}$, d'où le résultat.

Démonstration de la proposition 2.1. Soient $t_{0}, \ldots, t_{d}$ des variables. Il suffit de démontrer ce résultat dans le cas de $A=\mathbb{Z}\left[t_{0}, \ldots, t_{d}\right]$, pour le polynôme $P(x)=t_{d} x^{d}+\ldots+t_{0}$. Nous fixons un entier $n$ non nul, et un entier $k \in\{0, \ldots, n\}$, et nous effectuons la division euclidienne de $Q_{n, n}(x)$ par $Q_{k, n}(x)$ dans l'anneau $\mathbb{Q}\left(t_{0}, \ldots, t_{d}\right)[x]$. On a

$$
Q_{n, n}(x)=H_{k, n}(x) Q_{k, n}(x)+R_{k, n}(x),
$$

le degré en $x$ du polynôme $R_{k, n}$ étant inférieur à celui de $Q_{k, n}$. Les coefficients de $H_{k, n}(x)$ et de $R_{k, n}(x)$ sont des fonctions rationnelles des variables $t_{0}, \ldots, t_{d}$. Soit $B\left(t_{0}, \ldots, t_{d}\right) \in \mathbb{Z}\left[t_{0}, \ldots, t_{d}\right]$ le plus petit commun multiple des dénominateurs de ces coefficients, et posons

$$
\bar{H}_{k, n}(x)=B\left(t_{0}, \ldots, t_{d}\right) H_{k, n}(x)
$$

et

$$
\bar{R}_{k, n}(x)=B\left(t_{0}, \ldots, t_{d}\right) R_{k, n}(x)
$$

On a

$$
B\left(t_{0}, \ldots, t_{d}\right) Q_{k, n}(x)=\bar{H}_{k, n}(x) Q_{k, n}(x)+\bar{R}_{k, n}(x),
$$

l'égalité ayant lieu dans $\mathbb{Z}\left[t_{0}, \ldots, t_{d}, x\right]$. On spécialise maintenant les variables $t_{0}, \ldots, t_{d}$ en $a_{0}, \ldots, a_{d}$ dans $\mathbb{C}$, de façon que l'on ait $a_{d}$ non nul, $B\left(a_{0}, \ldots, a_{d}\right)$ non nul, que le discriminant des $P^{[i]}(x)-x, 1 \leq i \leq n$, après spécialisation, soit non nul (ce qui est possible d'après le résultat du lemme 2.7). Le polynôme spécialisé $\bar{P}$ vérifie alors les hypothèses du lemme 2.6. Par suite, le polynôme $\bar{R}_{k, n}(x)$ spécialisé est nul, pour tout choix d'une telle spécialisation. Le polynôme $\bar{R}_{k, n}(x)$ est donc le polynôme nul. Il en résulte que $Q_{k, n}(x)$ divise le polynôme $Q_{n, n}(x)$ dans $\mathbb{Q}\left(t_{0}, \ldots, t_{d}\right)[x]$.

Les coefficients de $Q_{k, n}(x)$ sont des éléments de l'anneau factoriel $\mathbb{Z}\left[t_{0}, \ldots, t_{d}\right]$ premiers entre eux dans leur ensemble. Pour le voir, il suffit de remarquer que le coefficient du terme de plus haut degré dans $Q_{k, n}(x)$ est une puissance de $t_{d}$, et que si on fait $t_{d}=0$, sans changer les autres variables $t_{0}, \ldots, t_{d-1}$, on trouve un polynôme non nul. Il existe donc au moins un coefficient de $Q_{k, n}(x)$ qui est premier à $t_{d}$, d'où le résultat. 
Il en résulte que $Q_{k, n}(x)$ divise $Q_{n, n}(x)$ dans l'anneau $\mathbb{Z}\left[t_{0}, \ldots, t_{d}\right][x]$, ce qui termine la démonstration de la proposition 2.1 dans le cas d'un degré $d \geq$ 2. Mais il est clair que l'on obtient le cas $d=1$ à partir du cas $d=2$ en faisant $t_{2}=0$ dans les formules précédentes, ce qui complète la démonstration.

On note dans toute la suite $b(n)$ le $n$-ième coefficient d'interpolation de la fonction entière $f(z)$ sur la suite $u(n)=P^{[n]}(z)$.

Les résultats suivants sont bien connus (cf. [GU], p. 34, formule 54, et p. 163-164, formules 122 et 123) :

Lemme 2.8. Le coefficient $b(n)$ est donné par les deux expressions suivantes :

(i) $\quad b(n)=\frac{1}{2 i \pi} \int_{|t|=R} \frac{f(t)}{\prod_{j=0}^{n}(t-u(j))} d t$

pour tout $R>\max \{|u(j)|: j=0, \ldots, n\}$,

(ii) $\quad b(n)=\sum_{k=0}^{n} \frac{f(u(k))}{\prod_{j \neq k, 0 \leq j \leq n}(u(k)-u(j))}$.

LEMME 2.9. On a la formule suivante:

$f(x)$

$=\sum_{k=0}^{n} b(k) \prod_{j=0}^{k-1}(x-u(j))+\frac{1}{2 i \pi} \prod_{j=0}^{n}(x-u(j)) \int_{|t|=R} \frac{f(t)}{(t-x) \prod_{j=0}^{n}(t-u(j))} d t$

pour $x \in \mathbb{C}$ et $R>\max \{|x|,|u(j)|: j=0, \ldots, n\}$.

Lemme 2.10. Soit $z \in \mathbb{Z}$ tel que pour $k \neq j$ on ait $P^{[k]}(z) \neq P^{[j]}(z)$. Alors la suite $\left|P^{[n]}(z)\right|$ tend vers l'infini si $n$ tend vers l'infini.

Démonstration. Si la suite $|u(n)|$ d'éléments de $\mathbb{N}$ est bornée, elle prend un nombre fini de valeurs et ne peut donc pas être injective.

Puisque le polynôme $P(x)$ est de degré $\geq 2$, il existe un réel positif $A$ tel que $x \in \mathbb{C},|x|>A$ implique $|P(x)|>2|x|$.

Soit alors $N$ un entier tel que $|u(N)|>A$. Une récurrence immédiate montre que $|u(N+m)| \geq 2^{m}|u(N)|$ pour tout $m \in \mathbb{N}$, d'où le lemme.

Lemme 2.11. Soit $z \in \mathbb{C}$, on suppose la suite $u(n)=P^{[n]}(z)$ injective. Il existe alors une constante $L>0$ telle que

$$
\log |u(n)|=L d^{n}+O(1)
$$

si $n$ tend vers l'infini. La constante $L$ n'est autre que la valeur au point $z$ de la fonction de Green du bassin d'attraction du point à l'infini pour le polynôme $P$. 
Démonstration. On a $u(n+1)=P(u(n))$, et on sait d'après le lemme 2.10 que la suite $|u(n)|$ tend vers l'infini. Posons $P(x)=q x^{d}+\ldots$ Il en résulte que, si $v(n)=\log |u(n)|$, on a la relation

$$
v(n+1)=d v(n)+\log |q|+\varepsilon(n)
$$

pour tout $n$ assez grand, la suite $\varepsilon(n)$ étant de limite nulle à l'infini. Posons $w(n)=v(n) / d^{n}$. On a alors

$$
w(n+1)=w(n)+\frac{\log |q|+\varepsilon(n)}{d^{n+1}},
$$

d'où, pour un entier convenable $N$,

$$
w(n)=w(N)+\sum_{k=N}^{n-1} \frac{\log |q|+\varepsilon(k)}{d^{k+1}} .
$$

On voit immédiatement que la série au second membre de l'égalité précédente converge, de sorte que $w(n)$ admet une limite finie, que nous notons $L$. De plus, on a l'égalité

$$
w(n)-L=\sum_{k=n}^{\infty} \frac{\log |q|+\varepsilon(k)}{d^{k+1}},
$$

d'où on déduit qu'il existe une constante positive $c$ telle que

$$
|w(n)-L| \leq c / d^{n+1} .
$$

En reprenant l'égalité $v(n)=w(n) d^{n}$, on voit que l'on a démontré l'estimation du lemme, à l'exception de la positivité de la constante $L$. Or, si celle-ci est négative ou nulle, il résulte de ce qui précède que la suite $|u(n)|$ ne tend pas vers l'infini si $n$ tend vers l'infini, ce qui démontre cette assertion. La valeur de la constante $L$ résulte immédiatement de la définition de la fonction de Green (cf. [BEGEMO], p. 283, formule 5).

Lemme 2.12. Pour $z \in \mathbb{Z}$ tel que la suite $u(n)=P^{[n]}(z)$ soit injective, on a

$$
\log \left|Q_{n, n}(z)\right|=n L d^{n}+O(n)
$$

si $n$ tend vers l'infini.

Démonstration. On a

$$
\log \left|Q_{n, n}(z)\right|=\sum_{0 \leq j<n} \log |u(n)-u(j)| .
$$

En prenant $n$ assez grand pour que $u(n) \neq 0$, il vient donc

$$
\log \left|Q_{n, n}(z)\right|=n \log |u(n)|+\sum_{0 \leq j<n} \log \left|1-\frac{u(j)}{u(n)}\right| .
$$


On a déjà vu que, pour un entier $N$ assez grand, on a une inégalité de la forme $|u(N+m)| \geq 2^{m}|u(N)|$, ce qui permet de majorer la somme au second membre de l'égalité précédente par une constante indépendante de $n$.

Le lemme résulte alors immédiatement du lemme 2.11.

LEMME 2.13. Soit $f(x)$ une fonction entière d'une variable complexe, et $z \in \mathbb{Z}$. On suppose que la suite $u(n)=P^{[n]}(z)$ est injective et que $f$ vérifie l'estimation

$$
\log |f|(R) \leq \frac{\log R \log \log R-\log R \log \log \log R}{\log d}-\mu \log R
$$

avec $\mu>-(\log \log d) / \log d$. Alors $f$ est somme de sa série de Newton aux points de la suite $u(n)$.

Démonstration. Nous allons majorer le reste

$$
R_{n}(x)=\frac{1}{2 i \pi} \prod_{j=0}^{n}(x-u(j)) \int_{|t|=R} \frac{f(t)}{(t-x) \prod_{j=0}^{n}(t-u(j))} d t
$$

et montrer que celui-ci tend vers zéro si $n$ tend vers l'infini, $x$ étant fixé. On a la majoration

$$
\left|R_{n}(x)\right| \leq \frac{\prod_{j=0}^{n}(|x|+|u(j)|) R|f|(R)}{(R-|x|) \prod_{j=0}^{n}(R-|u(j)|)},
$$

donc

$$
\begin{aligned}
\log \left|R_{n}(x)\right| \leq & \sum_{j=0}^{n} \log (|x|+|u(j)|)+\log |f|(R)+\log R \\
& -\log (R-|x|)-\sum_{j=0}^{n} \log (R-|u(j)|) .
\end{aligned}
$$

Nous prenons $R=\exp \left(n d^{n+1}\right)$, qui est supérieur à tous les $|u(j)|, 0 \leq j \leq n$, pourvu que $n$ soit pris assez grand. Compte tenu du lemme 2.11, il vient alors sans difficultés la majoration

$$
\log \left|R_{n}(x)\right| \leq \frac{L}{d-1} d^{n+1}+\log |f|(R)-n(n+1) d^{n+1}+O(n) .
$$

En utilisant l'hypothèse faite sur $|f|(R)$, il vient alors

$$
\log \left|R_{n}(x)\right| \leq-\left(\frac{\log \log d}{\log d}+\mu\right) n d^{n+1}+o\left(n d^{n}\right)
$$

et comme $\mu>-(\log \log d) / \log d$, ceci prouve le lemme.

Lemme 2.14. Soient b et $c$ deux réels positifs. Pour $t>b$, la fonction $\psi(x)=x(t-b \exp (c x))$ définie sur $[0, \infty[$ admet un unique maximum en 
un point $x_{0}(t)$, et la valeur de ce maximum, que nous notons $\phi(t)$, admet l'estimation

$$
\phi(t)=t \frac{\log t-\log \log t}{c}+O(t)
$$

quand t tend vers l'infini.

Démonstration. On a $\psi^{\prime}(x)=t-b(1+c x) \exp (c x)$, et la dérivée seconde $\psi^{\prime \prime}(x)=-b c \exp (c x)-b c(1+c x) \exp (c x)$ est toujours négative. La valeur en zéro de la dérivée est égale à $t-b$ qui est positif, et la dérivée tend vers $-\infty$ si $x$ tend vers l'infini. Ceci prouve la première assertion, avec de plus le fait que le point $x_{0}(t)$ vérifie $t=b\left(1+c x_{0}(t)\right) \exp \left(c x_{0}(t)\right)$; donc $x_{0}(t)$ est la valeur au point $t$ de la fonction réciproque de la fonction $b(1+c x) \exp (c x)$. En particulier, $x_{0}(t)$ tend vers l'infini si $t$ tend vers l'infini. En prenant le logarithme de l'égalité ci-dessus, on obtient

$$
c x_{0}(t)+\log x_{0}(t)+\log c+\log \left(1+\frac{1}{c x_{0}(t)}\right)+\log b=\log t .
$$

On met dans cette égalité $c x_{0}(t)$ en facteur dans le premier membre, on prend le logarithme; on obtient alors en tenant compte du fait que $x_{0}(t)$ tend vers l'infini,

$$
\log x_{0}(t)=\log \log t+O(1) .
$$

On reporte alors ceci dans la première égalité, d'où

$$
x_{0}(t)=\frac{\log t-\log \log t}{c}+O(1)
$$

On a

$$
\phi(t)=x_{0}(t)\left(t-b \exp \left(c x_{0}(t)\right)\right)=\frac{t c x_{0}(t)^{2}}{1+c x_{0}(t)},
$$

d'où

$$
\phi(t)=t\left(x_{0}(t)+O(1)\right)=t \frac{\log t-\log \log t}{c}+O(t),
$$

ce qui termine la démonstration du lemme.

Lemme 2.15. Soit $x \in \mathbb{C}$, de module assez grand, et $k \in \mathbb{N}$ non nul. On définit l'entier $N$ par les inégalités $\left|P^{[N+1]}(z)\right|>|x| \geq\left|P^{[N]}(z)\right|$. (Cet entier est bien défini car il résulte du lemme 2.11 que la suite $\left|P^{[n]}(z)\right|$ est croissante à partir d'un certain rang.) On a alors les majorations suivantes :

(i) pour $0 \leq k \leq N+1$,

$$
\log \prod_{j=0}^{k-1}\left|x-P^{[j]}(z)\right| \leq k \log |x|+c_{1},
$$


(ii) pour $k \geq N+2$,

$$
\log \prod_{j=0}^{k-1}\left|x-P^{[j]}(z)\right| \leq(N+1) \log |x|+\sum_{j=N+2}^{k-1} \log \left|P^{[j]}(z)\right|+c_{2},
$$

où $c_{1}$ et $c_{2}$ sont deux constantes positives.

Démonstration. On majore tout d'abord $\left|x-P^{[j]}(z)\right| \operatorname{par}|x|+$ $\left|P^{[j]}(z)\right|$. Dans le cas (i), on a alors comme majoration

$$
\log \prod_{j=0}^{k-1}\left(|x|+\left|P^{[j]}(z)\right|\right) \leq k \log |x|+\sum_{j=0}^{k-1} \log \left(1+\frac{\left|P^{[j]}(z)\right|}{|x|}\right) .
$$

Comme $\left|P^{[N]}(z)\right| \leq|x|$, il vient

$$
\log \prod_{j=0}^{k-1}\left(|x|+\left|P^{[j]}(z)\right|\right) \leq k \log |x|+\sum_{j=0}^{k-1} \log \left(1+\frac{\left|P^{[j]}(z)\right|}{\left|P^{[N]}(z)\right|}\right) .
$$

Soit $A$ un réel positif tel que pour tout $x \in \mathbb{C}$ tel que $|x|>A$ on ait $|P(x)| \geq 2|x|$, et $m$ un entier tel que $\left|P^{[j]}(z)\right|>A$ pour $j>m$. On a alors pour tout $j>m$ l'inégalité $\left|P^{[N]}(z)\right| \geq 2^{N-j}\left|P^{[j]}(z)\right|$. On en déduit que

$$
\sum_{j=0}^{k-1} \log \left(1+\frac{\left|P^{[j]}(z)\right|}{\left|P^{[N]}(z)\right|}\right) \leq \sum_{j=0}^{m} \log \left(1+\frac{\left|P^{[j]}(z)\right|}{\left|P^{[N]}(z)\right|}\right)+\sum_{i=0}^{\infty} \log \left(1+\frac{1}{2^{i}}\right)
$$

et le résultat en découle.

Nous passons maintenant au cas (ii). On a

$$
\log \prod_{j=0}^{k-1}\left(|x|+\left|P^{[j]}(z)\right|\right)=\log \prod_{j=0}^{N}\left(|x|+\left|P^{[j]}(z)\right|\right)+\log \prod_{j=N+1}^{k-1}\left(|x|+\left|P^{[j]}(z)\right|\right)
$$

et le premier terme de cette somme est, par le cas (i) que nous venons de démontrer, majoré par $(N+1) \log |x|+c_{1}$. D'autre part,

$\log \prod_{j=N+1}^{k-1}\left(|x|+\left|P^{[j]}(z)\right|\right)=\sum_{j=N+1}^{k-1} \log \left|P^{[j]}(z)\right|+\sum_{j=N+1}^{k-1} \log \left(1+\frac{|x|}{\left|P^{[j]}(z)\right|}\right)$.

En tenant compte du fait que $|x|<\left|P^{[N+1]}(z)\right|$, on montre comme dans l'étude du premier cas que le deuxième terme est majoré indépendamment de $N$, d'où le résultat.

III. Démonstration des résultats. D'après le lemme 2.13 et le fait que

$$
\frac{L}{d}-\frac{\log \log d}{\log d}>-\frac{\log \log d}{\log d},
$$


la fonction $f(x)$ est somme de sa série d'interpolation de Newton aux points $u(n)=P^{[n]}(z)$. Il suffit donc de démontrer que les coefficients d'interpolation $b(n)$ sont tous nuls à partir d'un certain rang. Il résulte du lemme 2.8(ii) et de la proposition 2.1 que les quantités $Q_{n, n}(z) b(n)$ sont des éléments de $\mathbb{Z}$. Le lemme 2.8(i) permet de majorer $b(n)$; on choisit de prendre dans ce lemme $R=R_{n}=\exp \left(n d^{n+1}\right)$. Les calculs sont analogues à ceux déjà faits dans la démonstration du lemme 2.13. On utilise le lemme 2.12 pour majorer $Q_{n, n}(z)$. On trouve comme majoration

$$
\log \left|Q_{n, n}(z) b(n)\right| \leq-\left(\lambda+\frac{\log \log d}{\log d}-\frac{L}{d}\right) n d^{n+1}+o\left(n d^{n}\right),
$$

ce qui, compte tenu de l'hypothèse faite sur $\lambda$, démontre le théorème.

Preuve de la proposition 2. Nous démontrons tout d'abord que $f(x)$ est une fonction entière de la variable complexe $x$. Posons

$$
W_{k}(x)=\frac{\prod_{j=0}^{k-1}\left(x-P^{[j]}(z)\right)}{\prod_{j=0}^{k-1}\left(P^{[k]}(z)-P^{[j]}(z)\right)} .
$$

Nous nous plaçons sous les hypothèses du lemme 2.15 et reprenons les mêmes notations. Pour $k>N+1$, on a, en utilisant (ii) du lemme 2.15 et le lemme 2.11,

$$
\log \left|W_{k}(x)\right| \leq(N+1) \log |x|+\sum_{j=N+2}^{k-1} \log \left|P^{[j]}(z)\right|-k \log \left|P^{[k]}(z)\right|+c_{3},
$$

où $c_{3}$ est une constante indépendante de $x$ et de $k$, puis

$$
\log \left|W_{k}(x)\right| \leq(N+1) L d^{N+1}+L \frac{d^{k}-d^{N+2}}{d-1}-k L d^{k}+c_{4} k,
$$

où la constante $c_{4}$ est indépendante de $x$ et de $k$. Posons $k=N+2+h$, avec $h$ entier naturel. On a

$$
\log \left|W_{k}(x)\right| \leq(N+2) L d^{N+1} \omega(N, h)-h L d^{h}+c_{4} h,
$$

où on a posé

$$
\omega(N, h)=-d^{h+1}+\frac{d^{h+1}}{(d-1)(N+2)}+\frac{N+1}{N+2}+\frac{c_{4}}{L d^{N+1}} .
$$

Si l'on a pris $N$ assez grand, la quantité $\omega(N, h)$ est négative pour tout $h \in \mathbb{N}$, donc on a la majoration

$$
\log \left|W_{k}(x)\right| \leq-h L d^{h}+c_{4} h
$$

et cette majoration montre que la série $W_{k}(x)$ est convergente. Il en résulte que la fonction $f(x)$ est bien une fonction entière de la variable $x$. 
Nous allons maintenant majorer la croissance de $f(x)$. Il résulte de ce qui précède que l'on a, pour $|x|$ assez grand,

$$
\sum_{k=N+2}^{\infty}\left|W_{k}(x)\right| \leq \sum_{h=0}^{\infty} \exp \left(-h L d^{h}+c_{5} h\right)
$$

et cette dernière quantité est une constante indépendante de $x$. Il reste à majorer $\sum_{k=0}^{N+1}\left|W_{k}(x)\right|$. Par le lemme 2.15 et le lemme 2.11, on a pour $k \in\{1, \ldots, N+1\}$,

$$
\log \left|W_{k}(x)\right| \leq k \log |x|-k L d^{k}+c_{6} k
$$

pour une constante positive $c_{6}$. On applique le résultat du lemme 2.14 avec $t=\log |x|+c_{6}, b=L$ et $c=\log d$. Un calcul immédiat montre alors que

$$
\log \left|W_{k}(x)\right| \leq \frac{\log |x| \log \log |x|-\log |x| \log \log \log |x|}{\log d}+O(\log |x|) .
$$

Par suite,

$$
\begin{aligned}
& \sum_{k=0}^{N+1}\left|W_{k}(x)\right| \\
& \leq(N+2) \exp \left(\frac{\log |x| \log \log |x|-\log |x| \log \log \log |x|}{\log d}+O(\log |x|)\right)
\end{aligned}
$$

et comme $\log N$ est un $O(\log |x|)$, l'estimation sur la croissance de $f(x)$ est démontrée. Enfin, on a $f(z)=f\left(P^{[0]}(z)\right)=1$, et comme les coefficients d'interpolation de $f(x)$ sur la suite $P^{[n]}(z)$ sont égaux à $1 / Q_{n, n}(z)$, la formule (ii) du lemme 2.8 permet de démontrer par récurrence que $f\left(P^{[n]}(z)\right) \in$ $\mathbb{Z}$ pour tout $n \in \mathbb{N}$. Comme il est clair que $f(x)$ n'est pas un polynôme, nous avons terminé la démonstration de la proposition 2 .

Démonstration $\mathrm{d} u$ corollaire 3 . On prend $P(x)=x^{d}$; on a $L(z, P)=\log |z|$, et le théorème 1 s'applique immédiatement.

Démonstration $\mathrm{du}$ corollaire 4 . On calcule facilement $\omega=$ $\left(z+\sqrt{z^{2}-4}\right) / 2$. Posons $\omega=\exp (i \theta)$ avec $\theta \in \mathbb{C}$. Soit $P(x)=x^{2}-2$. On a immédiatement que $P(2 \cos (\theta))=2 \cos (2 \theta)$, et par une récurrence facile que $P^{[n]}(z)=P^{[n]}(2 \cos (\theta))=2 \cos \left(2^{n} \theta\right)=\omega^{2^{n}}+\omega^{-2^{n}}$. On peut donc appliquer le théorème 1 . Il vient que

$$
\log \left|P^{[n]}(z)\right|=2^{n} \log |\omega|+O(1) ;
$$

on en déduit que $L(z, P)=\log (|\omega|)=\log \left|\frac{z+\sqrt{z^{2}-4}}{2}\right|$, d'où le résultat. 


\section{Références}

[BEGEMO] D. Bessis, J. Geronimo et P. Moussa, Ensembles de Julia et propriétés de localisation des familles itérées d'entiers algébriques, C. R. Acad. Sci. Paris 299 (1984), 281-284.

[GU] A. O. Guelfond, Calcul des différences finies, Dunod, Paris, 1963.

[PO] G. Pólya, Ueber ganzwertige ganze Funktionen, Rend. Circ. Mat. Palermo 40 (1915), 1-16.

DÉPARTEMENT DE MATHÉMATIQUES

UNIVERSITÉ DE CAEN

ESPLANADE DE LA PAIX

14032 CAEN CEDEX, FRANCE

Reçu le 2.11.1993

et révisé le 24.2.1994 